International Journal of Wireless \& Mobile Networks (IJWMN) Vol. 7, No. 4, August 2015

\title{
THE SHADOWING PROPAGATION MODEL IN UNMANNED AERIAL VEHICLE NETWORKS
}

\author{
Jun Peng \\ Electrical Engineering Department \\ University of Texas - Rio Grande Valley \\ Edinburg, Texas, USA
}

\begin{abstract}
Unmanned aerial vehicles (UAVs) have become very popular recently for both civil uses and potential commercial uses, such as law enforcement, crop survey, grocery delivery, and photographing, although they were mainly used for military purposes before. Researchers need the help of simulations when they design and test new protocols for UAV networks because simulations can be done for a network of a size that a test bed can hardly approach. In the simulation of an UAV network it is important to choose a radio propagation model for the links in the network. We study the shadowing radio propagation model in this paper and compare it with the free space model, both of which are available in the ns 2 network simulation package. We also show how the choice of the parameters of the shadowing model would impact on the network performance of a UAV network.
\end{abstract}

\section{KEYWORDS}

The shadowing model, propagation models, unmanned aerial vehicles (UAV), network Simulation

\section{INTRODUCTION}

Unmanned aerial vehicles had been mainly used in military applications before, but they became popular in recent years in both civilian and commercial applications, as hardware and software became more advanced and cheaper. For example, people started to use UAVs to film subjects from perspectives that they could hardly imagine or reach before due to the high cost of having an access to a plane or helicopter. Other advantages of using UAVs in such an application include safety, efficiency, and environmental friendliness. UAVs have also been used for law enforcement, crowd management, surveillance, search and rescue, and scientific research [1].

It will become inevitable that UAVs will need to communicate with each other for purposes of coordination and collaborations in the future, as they become more and more popular in civilian and commercial applications. One example is that UAVs will likely need some type of coordination in the future to avoid collisions among themselves. Another example is that applications requiring a large amount of UAVs working together dynamically, such as doing a constant surveillance on a large geographical area, will also need the UAVs to communicate with each other effectively. 
Researchers have been investigating topics at all layers of UAV networks to enable UAVs for networking. Asadpour et al. [2] studied the performance of IEEE 802.11n interfaces in UAV networks and they found that $802.11 \mathrm{n}$ performed poorly in highly mobile scenarios. Cai et cal. [3] proposed a medium access control (MAC) protocol for UAV ad-hoc networks with full-duplex radios. They proposed a token based technique to update information in the network for efficiently handling the high mobility of UAVs. Ollero et al. [4] presented a platform that enables autonomous aerial vehicles to cooperate with ground wireless sensor-actuator networks. Alshbatat et al. [5] used the approach of cross layer design to deal with the high dynamics in UAV networks. Alshbatat et al. [6] also proposed an adaptive medium access control protocol for UAV networks using directional antennas. Gu et al. [7] proposed a routing scheme for multi-layer ad hoc networks that use UAVs in their backbones. Gu et al. [8] also presented a multiple access protocol for such type of network.

When UAV networks of large scales are needed in testing communication and networking protocols, simulations are usually the only practical way that would be available to most researchers. One important choice in simulating an UAV network is the radio propagation model used in the network. There are several radio propagation models that are available in the ns- 2 network simulation package [9]. Although there are studies on some of these radio propagation models for vehicular networks [2], they in general focus on terrestrial networks. In this paper we investigate the shadowing model in UAV networks and show how the choice of its parameters may have an impact on the performance of the simulated network. We also compare the shadowing model with the free space propagation model in the paper.

The rest of the paper is organized as follows. We introduce the the shadowing radio propagation model and the free space loss model in Section 2. We present in Section 3 numerical results on these two models using simulations. We finally make our conclusions in Section 4.

\section{THE SHADOWING RADIO PROPAGATION MODEL}

Before we introduce the shadowing model in this section we first introduce the free space propagation model because it may be used to calculate the reference power in the shadowing model. Besides, we will compare the free space loss model with the shadowing model in our simulations.

The free space radio propagation model assumes, as its name indicates, that the transmitter and the receiver are in free space without obstacles. The propagation formula is as follows [10]:

$$
P_{r}=P_{t} \frac{A_{r} A_{t}}{d^{2} \lambda^{2}}
$$

where $P_{r}$ is the signal power at the receiving antenna, $P_{t}$ is the signal power at the transmitting antenna, $A_{r}$ is the effective area of the receiving antenna, $A_{t}$ is the effective area of the transmitting antenna, $d$ is the distance between the transmitting antenna and the receiving antenna, and $\lambda$ is the wavelength of the signal.

The effective area of an isotropic antenna is $\lambda / 4 \pi$. Equation 1 thus becomes the following for isotropic antennas:

$$
P_{r}=P_{t} \frac{\lambda^{2}}{(4 \pi d)^{2}}
$$


Equation 1 becomes the following when the transmitting antenna and the receiving antenna have an antenna gain that is not equal to 1 :

$$
P_{r}=P_{t} G_{t} G_{r} \frac{\lambda^{2}}{(4 \pi d)^{2}}
$$

where $G_{t}$ is the antenna gain of the transmitting antenna and $G_{r}$ is the antenna gain of the receiving antenna.

As noted in [10], Equations 1 to 3 only apply under the following condition:

$$
d \geq \frac{2 a^{2}}{\lambda}
$$

Where $a$ is the largest linear dimension of either of the antennas.

Because of multipath propagation effects, in reality the received power at a receiving antenna is not a deterministic function of the communication distance as shown in the free space loss model. Instead, the received power fluctuates randomly versus time. The shadowing model [11] better reflects this characteristic of the received signal power in reality than the free space loss model introduced earlier. The path loss is usually expressed in $\mathrm{dB}$ as follows in the the shadowing model:

$$
\left[P_{r}(d)\right]_{d B}=\left[P_{r}\left(d_{0}\right)\right]_{d B}-10 \beta \log \left(\frac{d}{d_{0}}\right)+X_{d B}
$$

where $d_{0}$ is a reference distance, $\beta$ is the path loss exponent, $X_{d B}$ is a Gaussian random variable having a zero mean and a standard deviation of $\delta_{d B}$. The typical values of $\beta$ and $\delta$ are shown in Tables 1 and 2, respectively.

Table 1 Typical value of $\beta$ in different environment

\begin{tabular}{|c|c|}
\hline Environment & $\beta$ \\
\hline Outdoor, free space & 2 \\
\hline Outdoor, shadowed urban area & $2.7-5$ \\
\hline In building, line-of-sight & $1.6-1.8$ \\
\hline In building, obstructed & $4-6$ \\
\hline
\end{tabular}

Table 1 Typical value of $\delta$ in different environment

\begin{tabular}{|c|c|}
\hline Environment & $\delta$ \\
\hline Outdoor & $4-12$ \\
\hline Office, hard partition & 7 \\
\hline Office, soft partition & 9.6 \\
\hline Factory, line-of-sight & $3-6$ \\
\hline Factory, obstructed & 6.8 \\
\hline
\end{tabular}




\section{SIMULATION RESULTS}

In this section we study the shadowing model in UAV networks using the ns 2 simulation package. We also compare this model with the free space propagation model in this section. The simulation package ns 2 does not support simulations of three dimensional networks readily. We modified the code for simulating UAV networks.

The test bed used in our simulations is shown in Figure 1, which has the same size of 500 meters for its width, length, and height. UAVs fly freely in this defined space following the random waypoint model. The UAVs in the test bed use the IEEE 802.11 air interface and default settings are used in the simulations. For traffic, each UAV sets up a CBR connection with another UAV at a random time in the duration of each simulation. A CBR source has a packet rate of 10 packets per second and the size of each packet is 512 bytes. The UAVs use the DSR routing protocol [12] in the test bed.

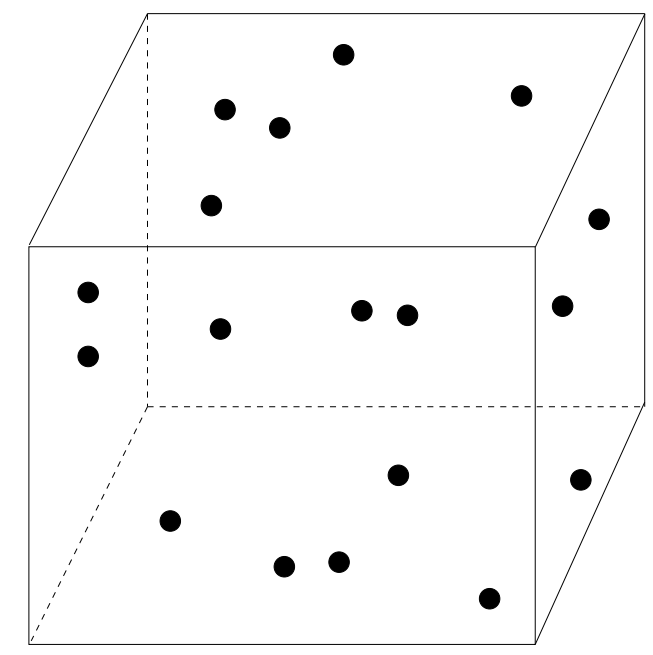

Figure 1 The simulated test bed

In our simulations we tested the shadowing model with varying node densities in the test bed and compared it with the free space propagation model. In particular, for each model we varied the number of nodes in the test bed from 10 to 50 in our simulations of UAV networks. In each simulation we gathered data to calculate the average throughput and the average packet loss rate of the CBR flows in the network. We also gathered data on the packet delay distributions in the network. The simulation results comparing the two models are shown in Figures 2 to 9.

As shown in Figure 2, the average flow throughput in the network decreases as the node density increases in the network. This decrease of flow throughput is mainly caused by two factors. One is that the total traffic rate is higher in the network when there are more nodes in the network because each node starts a CBR flow at a random time in each simulation. The other factor is that the contention at the medium access control (MAC) layer is also higher when there are more nodes in the network. The throughput results are also corroborated by the packet loss rate shown in Figure 3. As shown in Figure 3, a higher node density in the network results in a higher packet loss rate in the network. 
As also shown in Figures 2 and 3, both the flow throughput trend and the packet loss rate trend are consistent for the two propagation models. However, the throughput for the shadowing model is significantly lower as compared with that of the free space model. The difference can reach about thirty percent when the node density is low. When the node density is high, however, the difference is smaller because the network becomes very congested in such a case and lots of packet are dropped for both models. Similar observations can be made in Figure 3 for packet losses for the two models.

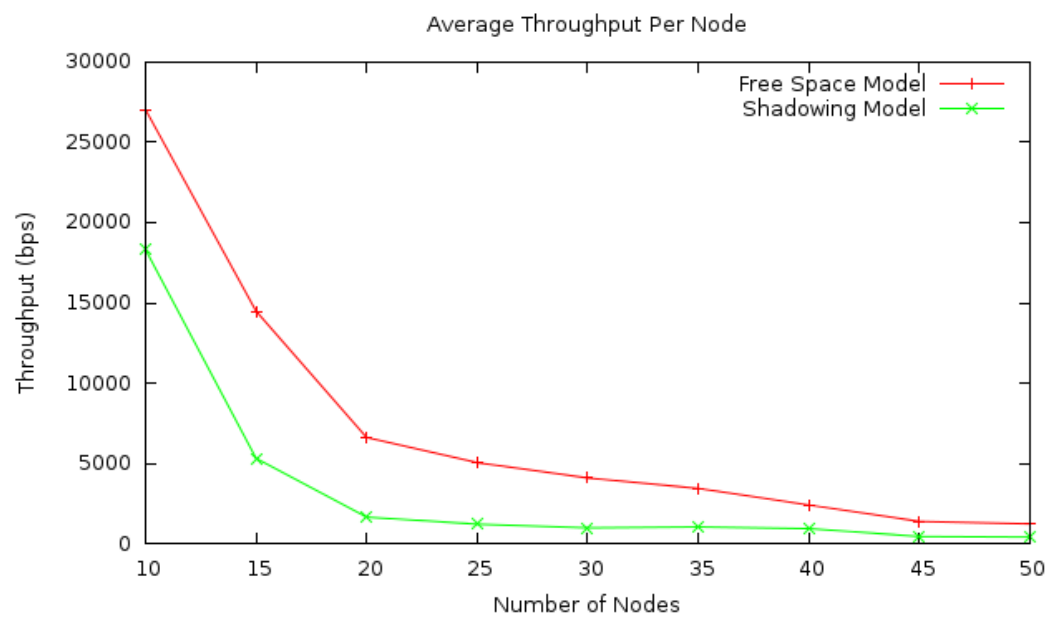

Figure 2. The average flow rate for the two propagation models

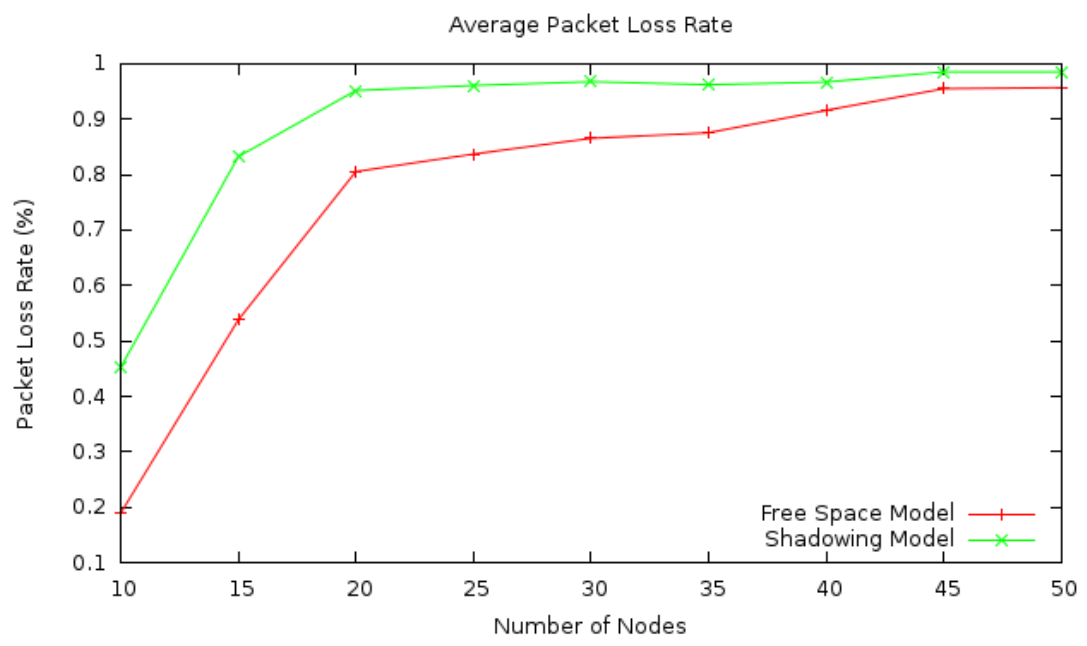

Figure 3. The average packet loss rate for the two propagation models

The packet delay data shown in Figures 4 and 5 for the case of ten nodes in the test bed also corroborate the results shown in earlier figures for the two models. As shown by Figures 4 and 5, most packets have a delay within 0.2 second for the free space propagation model. For the shadowing model, however, much more packets have a delay beyond 0.2 second. These packet delay results are consistent with the throughput and loss results shown earlier. 


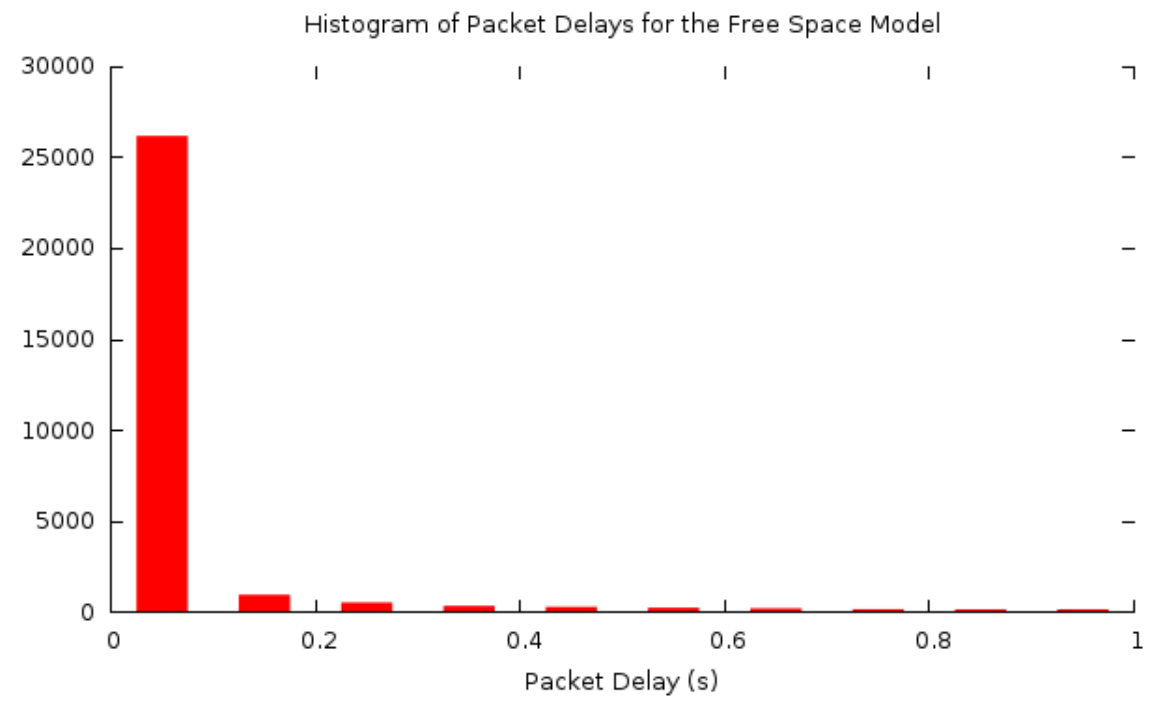

Figure 4. The histogram of the packet delays for the free space loss model

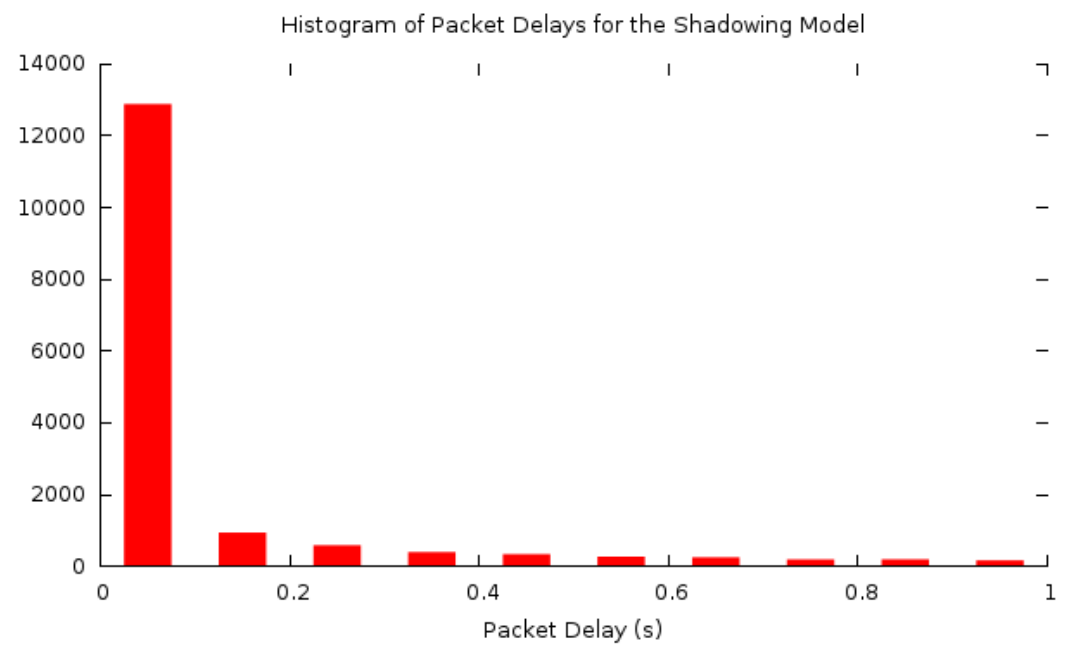

Figure 5. The histogram of the packet delays for the shadowing loss model

After showing the results comparing the shadowing model with the free space model, we now present more results on the shadowing model. Figures 6 and 7 show the average flow rate and the average packet loss rate, respectively, in the test bed of ten nodes for the model. The horizontal axis in these two figures is the $\delta$ parameter of the shadowing model, which reflects the severity of the multipath effects on the links. As shown in Figure 6, the average flow throughput decreases as $\delta$ increases. In particular, when $\delta$ increases from 2 to 12 the throughput decreases from above 18000 bps to below 8000 bps. The packet loss results in Figure 7 show results that are consistent with the throughput results. A higher $\delta$, which means higher multipath effects, results in a lower average flow throughput and a higher average packet loss rate. 


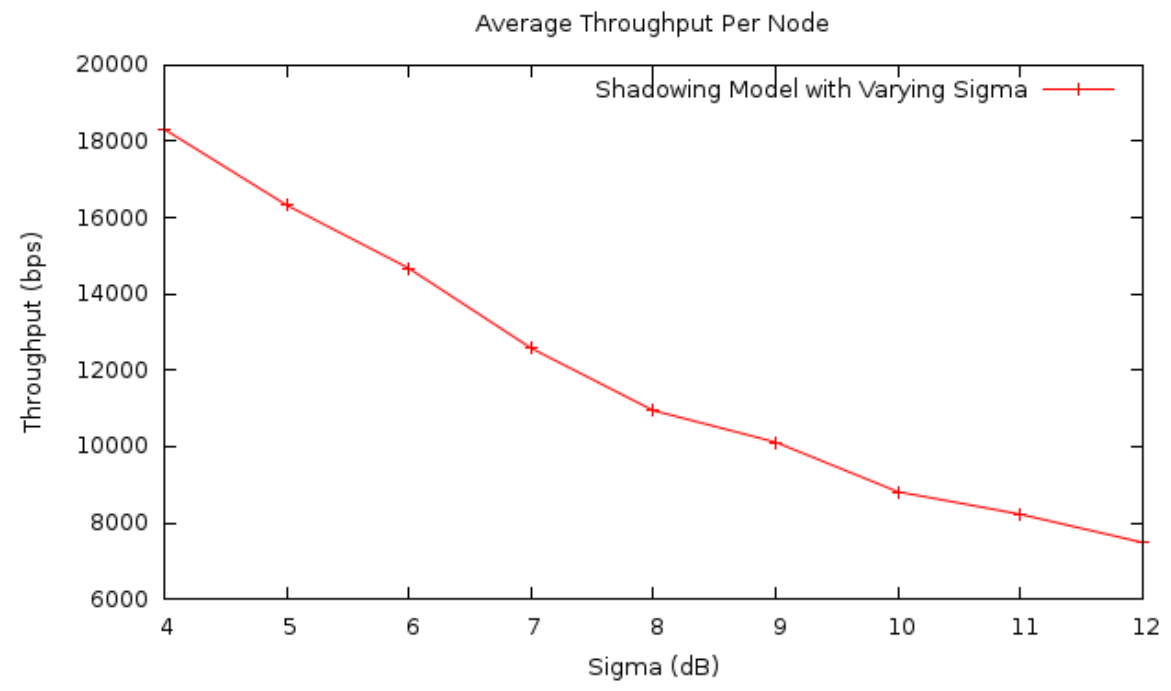

Figure 6. The average flow rate for the shadowing model with varying $\delta$

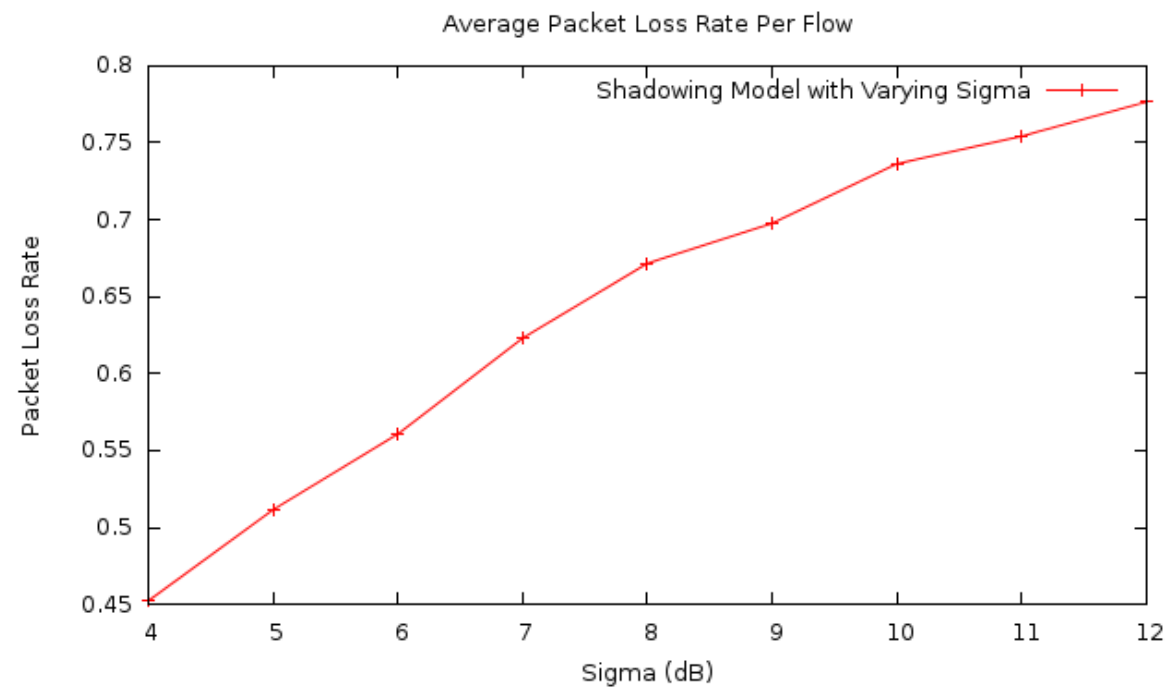

Figure 7. The average packet loss rate for the shadowing model with varying $\delta$

Figures 8 and 9 present the average flow rate and the average packet loss rate, respectively, versus the $\beta$ parameter of the shadowing model, which reflects the severity of the path loss. As shown in Figure 8, the average flow throughput decreases rapidly as $\beta$ increases even slowly. In particular, when $\beta$ increases from 2 to 2.6 the throughput decreases from above 18000 bps to almost zero bps. The packet loss results presented in Figure 9 shows results that are also consistent with the throughput results. A higher $\beta$, which means a higher path loss, results in a lower average flow throughput and a higher average packet loss rate. When $\beta$ reaches 2.6 in the test bed, the packet loss rate reaches almost one hundred percent. 


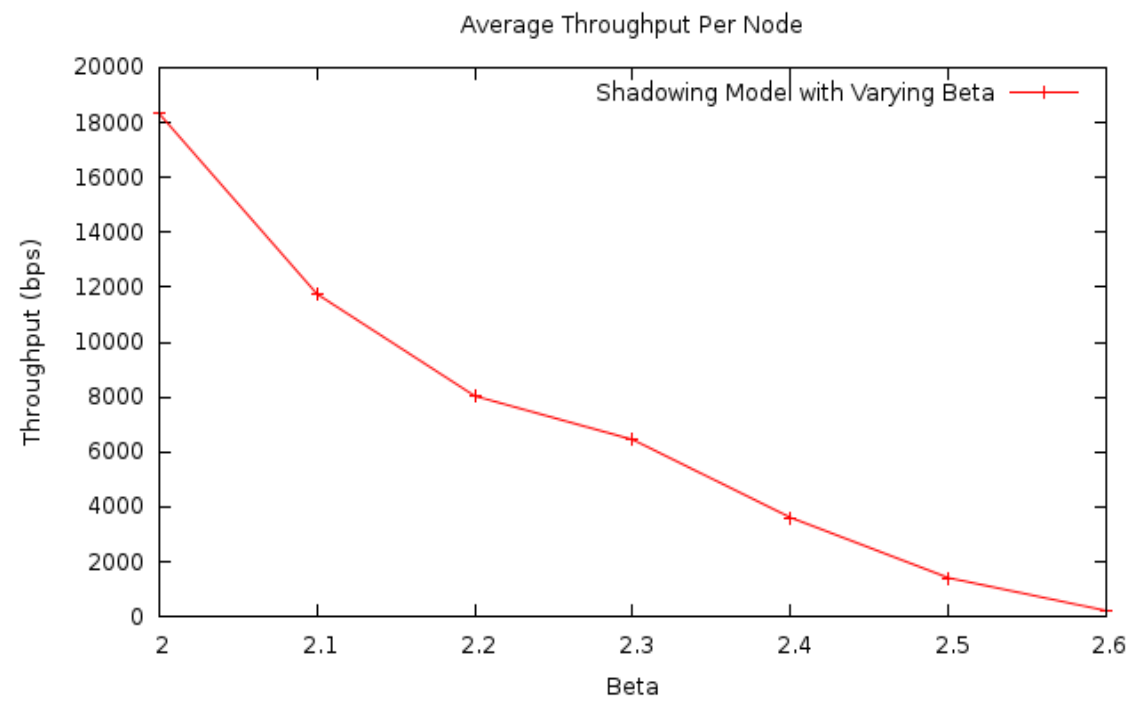

Figure 8 . The average flow rate for the shadowing model with varying $\beta$

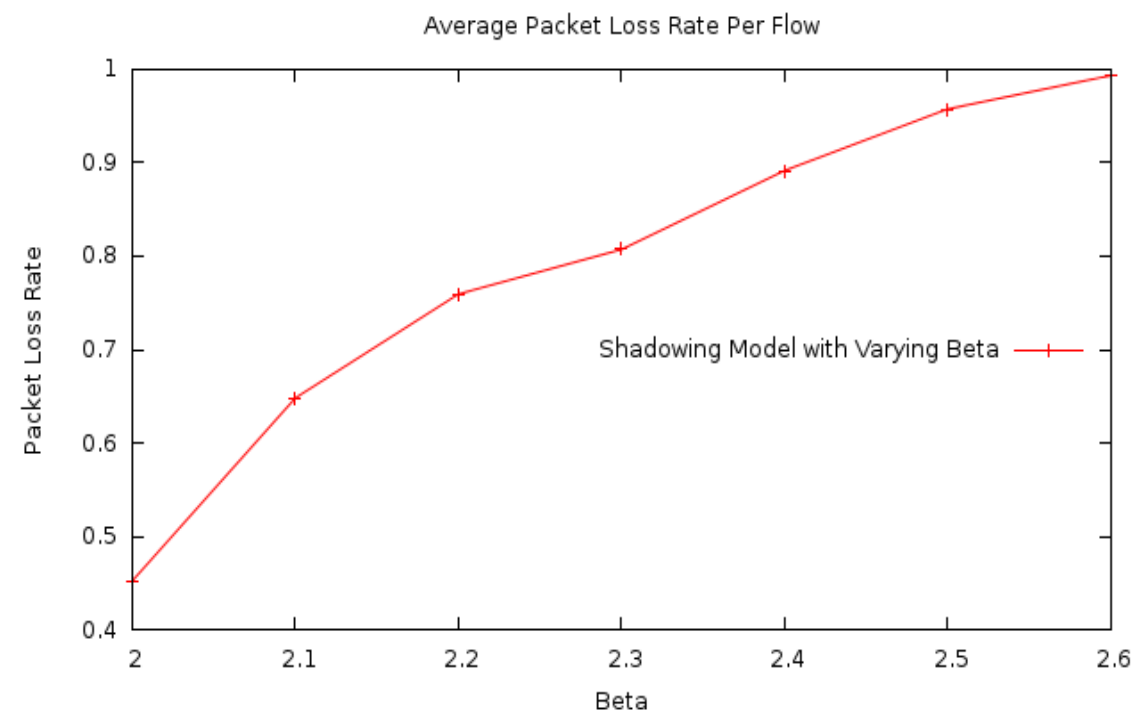

Figure 9. The average packet loss rate for the shadowing model with varying $\beta$

In summary, the choice of a radio propagation model in the simulation of an UAV network does have a significant impact on the performance of the network. Moreover, the choice of the parameters of a model are also important in simulating a UAV network, as shown by our study of the shadowing model with varying parameters. We also found, however, that even the shadowing model had simulation results very different from what the free space model had in each specific case of simulation, the trend of the simulation results observed as a whole did show consistency for the two models that we studied. 
International Journal of Wireless \& Mobile Networks (IJWMN) Vol. 7, No. 4, August 2015

\section{CONCLUSIONS}

We investigated the shadowing radio propagation model in UAV networks using the ns 2 network simulation package. Our investigation shows that the shadowing model may generate simulation results that are significantly different from those of the free space loss model in each specific case of simulation. Particularly, the flow throughput, the packet loss rate, and the packet delays consistently show much lower performance for the shadowing model than for the free space model. However, our results also show that when the simulation results are observed as a whole, the trend of flow throughput, packet loss rate, and packet delays is consistent for the two models. We also found that the choice of the parameters of the shadowing propagation model is significant in terms of network performance. Our conclusion is that the choice of a radio propagation model and its parameters in simulating an UAV network does have significant impact on the simulation results for each individual case of simulation, although the choice does not affect the interpretation of the data when performance trend is the only concern of investigation.

\section{ACKNOWLEDGEMENTS}

The authors would like to thank everyone who offered support to this project and the publication of its results.

\section{REFERENCES}

[1] T. H. Cox, C. J. Nagy, M. A. Skoog, and I. A. Somers, "Civil UAV Capability Assessment," NASA, 2004.

[2] M. Asadpour, D. Giustiniano, K. A. Hummel, and S. Heimlicher, "Characterizing 802.11N Aerial Communication," in Proceedings of the Second ACM MobiHoc Workshop on Airborne Networks and Communications, 2013, pp. 7-12.

[3] Y. Cai, F. Yu, J. Li, Y. Zhou, and L. Lamont, "Medium Access Control for Unmanned Aerial Vehicle (UAV) Ad-Hoc Networks With Full-Duplex Radios and Multipacket Reception Capability," Vehicular Technology, IEEE Transactions on, vol. 62, pp. 390-394, Jan 2013.

[4] A. Ollero, M. Bernard, M. La Civita, L. van Hoesel, P. Marron, J. Lepley, and E. de Andres, "AWARE: Platform for Autonomous self-deploying and operation of Wireless sensor-actuator networks cooperating with unmanned AeRial vehiclEs," in Safety, Security and Rescue Robotics, 2007, IEEE International Workshop on, Sept 2007, pp. 1-6.

[5] A. Alshbatat and L. Dong, "Adaptive MAC protocol for UAV communication networks using directional antennas," in Networking, Sensing and Control (ICNSC), 2010 International Conference on, April 2010, pp. 598-603.

[6] A. Alshbatat and L. Dong, "Cross layer design for mobile Ad-Hoc Unmanned Aerial Vehicle communication networks," in Networking, Sensing and Control (ICNSC), 2010 International Conference on, April 2010, pp. 331-336.

[7] D. Gu, G. Pei, H. Ly, M. Gerla, and X. Hong, "Hierarchical routing for multi-layer ad-hoc wireless networks with UAVs," in MILCOM 2000, vol. 1, 2000, pp. 310-314.

[8] D. Gu, H. Ly, X. Hong, M. Gerla, G. Pei, and Y.-Z. Lee, "C-ICAMA, a centralized intelligent channel assigned multiple access for multi-layer ad-hoc wireless networks with UAVs," in Wireless Communications and Networking Conference, 2000, IEEE, vol. 2, 2000, pp. 879-884.

[9] The network simulator - ns-2. [Online]. Available: http://www.isi.edu/nsnam/ns/

[10] H. T. Friis, "A note on a simple transmission formula," in Proceedings of the I.R.E. and Waves and Electrons, 1946, pp. 254-256.

[11] T. S. Rappaport, "Wireless Communications, Principles and Practice," Prentice Hall, 1996.

[12] D. B. Johnson, D. A. Maltz, and Y.-C. Hu, "The Dynamic Source Routing Protocol for Mobile Ad Hoc Networks (DSR)," IETF Interet draft,draft-ietf-manet-dsr-10.txt, July 2004. 
International Journal of Wireless \& Mobile Networks (IJWMN) Vol. 7, No. 4, August 2015

\section{Authors}

Jun Peng received his Ph.D. degree from the Department of Electrical, Computer \& Systems Engineering at Rensselaer Polytechnic Institute, Troy, New York, United States. He is currently an associate professor at the Electrical Engineering Department of University of Texas - Rio Grande Valley, Edinburg, Texas, United States. His research interests are in computer and communication networks. 